\title{
Factores de riesgo de la enfermedad periodontal: factores genéticos
}

\author{
RIOBOO CRESPO M* \\ BASCONES A**
}

Rioboo Crespo M, Bascones A. Factores de riesgo de la enfermedad periodontal: factores genéticos. Av Periodon Implantol. 2005; 17, 2: 69-77.

\begin{abstract}
RESUMEN
Hoy en día y tras numerosos estudios epidemiológicos, se acepta el concepto de la existencia de determinados factores de riesgo que van a modular la susceptibilidad o resistencia del hospedador a padecer enfermedad periodontal, por lo tanto, en el desarrollo van a intervenir varias causas considerándose dicha patología de etiología multifactorial. De este modo, las enfermedades periodontales son producidas por una interacción de un agente microbiano único o múltiple considerado como el factor etiológico primario necesario pero no suficiente, un huésped más o menos susceptible y unos factores ambientales que influyen sobre ambos.
\end{abstract}

La importancia de la herencia y la genética para el conocimiento de la etiopatogenia de la enfermedad periodontal y para la práctica clínica en general fueron destacadas desde muy temprano, pero las complejas interacciones que ocurren entre los mecanismos de respuesta del hospedador y la acción de los microorganismos patógenos han hecho que las aclaraciones sobre el papel de los factores genéticos en la enfermedad periodontal sean más difíciles. Aun así, la influencia de los factores genéticos en la periodontitis parece ser diferente para los distintos tipos de enfermedad periodontal y ha sido estudiada en cada una de las periodontitis definidas.

En general, se considera que hay suficiente base científica a favor de la presencia de factores genéticos en la aparición de periodontitis agresivas. En las periodontitis crónicas, en cambio, la evidencia de la participación genética es menos manifiesta. Numerosos estudios ponen en evidencia que existe una asociación entre las periodontitis y una variación genética de determinados genes (polimorfismos) que codifican diferentes citoquinas proinflamatorias y mediadores involucrados en la etiopatogénia de la enfermedad periodontal como la IL-1,IL4, IL-10, TNF, PGE2. El fenotipo HLA( Human Leukocite Antigen), también ha sido investigado como posible factor de resistencia y susceptibilidad de la enfermedad periodontal así como los polimorfismos del gen de la vitamina D, del receptor fMLP, del receptor FcIIIb de los neutrófilos, del receptor FcүRII y de la N-acetyltransferasa (NAT2).

\section{PALABRAS CLAVE}

Enfermedades periodontales. Periodontitis. Factores de riesgo. Factores genéticos.

Aceptado para publicación: Abril 2005

* Licenciada en Odontología. Becaria de Formación de Profesorado Universitario.

** Catedrático del Departamento III Estomatología.

Facultad de Odontología UCM 


\section{INTRODUCCIÓN}

Hoy en día y tras numerosos estudios epidemiológicos, se acepta el concepto de la existencia de determinados factores de riesgo que van a modular la susceptibilidad o resistencia del hospedador a padecer enfermedad periodontal, por lo tanto, en su desarrollo van a intervenir varias causas considerándose dicha patología de etiología multifactorial.

En el caso de la mayoría de las enfermedades infecciosas como la enfermedad periodontal, se conoce que la presencia de microorganismos no siempre va acompañada por los signos o síntomas característicos de este trastorno. Así, el germen por si sólo no es suficiente para causar una presencia patológica sino que el desarrollo de la enfermedad puede depender de otros factores.

El término factor de riesgo se refiere a condiciones o características que se asocian fuertemente con individuos o grupos que tienen una determinada enfermedad en contraste con los que no la tienen.

\section{CRITERIOS DE CAUSALIDAD}

Ante la imperfección de los métodos utilizados por los investigadores para conocer la realidad (las causas de una enfermedad) se recurre a una serie de criterios a los que clásicamente se les ha aplicado un claro matiz de probabilidad y que nos ayudan a definir una variable como factor de riesgo. Los más conocidos son los de Hill(1965) que constituyen un resumen de los elaborados por otros epidemiólogos:

1-Fuerza de la asociación: Medida a través del riesgo relativo (RR). Cuanto más alejado está el $R R$ de la unidad más fuerte se considera la asociación.

2-Consistencia: Repetición de la asociación en diferentes lugares, personas, circunstancias etc. Influyen los métodos de investigación que se ha utilizado y los errores aleatorios.

3-Especificidad: Plantea una relación biunívoca exclusiva.

4-Precedencia temporal de la causa: La causa debe preceder al efecto.

5-Gradiente biológico: Asume que todos los fenómenos siguen una relación creciente o decreciente uniforme con la cantidad de exposición antes de llegar a la saturación del efecto.
6-Plausibilidad biológica: Depende del grado de conocimiento biológico existente.

7-Coherencia: Criterio asociado al anterior, asume la no conflictividad con los hechos de la historia natural y de la biología de la enfermedad en el momento presente.

8-Evidencia Experimental: Disminución o desaparición del efecto cuando se suprime la causa.

9-Analogía: Valoración de una relación por similitud con otras.

Mas tarde se incluyeron los criterios de: "Probabilidad", que hace referencia a la significación estadística, "Cumplimiento de las predicciones", que consiste en demostrar que los hechos derivados de la asociación se producen en la realidad y "Ser comprobable" (1).

No todos estos criterios son esenciales para definir una variable como factor de riesgo. Su presencia ayuda, pero su ausencia no elimina una posible relación. Solo tres hechos parecen fundamentales.

- La existencia de una asociación

- La ausencia de ambigüedad temporal

- Que la asociación observada no sea debida enteramente a errores (azar, sesgo de confusión, sesgo de selección, sesgo de información).

Dichos factores se identifican en base a estudios epidemiológicos y pueden recibir diferentes denominaciones en función de su nivel de causalidad.

Se establece una jerarquía de la evidencia para los distintos factores de riesgo en relación con los diferentes tipos de estudios epidemiológicos que los sustentan. Así, el análisis de una determinada variable como factor de riesgo sigue los pasos siguientes (2):

- Primero se sugiere una posible relación entre determinados factores y la enfermedad basándose en informes de casos, anécdotas, etc.

- Se establece una asociación entre el factor y la enfermedad mediante estudios casos control y transversales.

- Le siguen los estudios longitudinales que aportan una fuerte evidencia de que el posible factor es un verdadero factor de riesgo, estableciéndose una secuencia temporal.

- Finalmente, el análisis de riesgo debe completarse mediante ensayos controlados aleatorios. 


\section{VALORACIÓN DEL RIESGO EN LA ENFERMEDAD PERIODONTAL}

El riesgo de una enfermedad es una proporción que indica la probabilidad de que ocurra un determinado suceso en un periodo de tiempo o edad determinados. El término riesgo lleva implícito la presencia de uno o más factores que incrementan dicha probabilidad. Contamos con diferentes procedimientos 0 métodos para evaluar el riesgo, los más utilizados son: el riego relativo ( $R R$ ) y y/o la odds ratio (OR).

El riesgo relativo es la razón del riesgo de enfermar entre los individuos expuestos a determinado factor patógeno, dividido por el riesgo de enfermar entre los individuos no expuestos a dicho factor. Cuando no se conoce la incidencia como ocurre en el caso de estudios de "casos y controles", la estimación del riego relativo se realiza calculando la OR (razón de productos cruzados) que se aproxima estrechamente al autentico valor del $R R$, sobre todo cuando la frecuencia de la enfermedad es baja. No existe el término equivalente en castellano en la traducción de "odds"(algunos utilizan "ventajas" o "oportunidades") pero ésta se define como el cociente entre la proporción de ocurrencia de un evento y la proporción de no ocurrencia. La odds ratio (OR) se define entonces como la razón o cociente entre dos odds.

Es necesario, antes de describir los diferentes factores que condicionan el nivel de riesgo de padecer enfermedad periodontal, definir varios conceptos que se manejan en la determinación del riesgo de enfermedad.

El riesgo puede ser identificado por diferentes términos: Factores de riesgo, determinantes de riesgo, indicadores de riesgo y predictores de riesgo (3).

-Factor de riesgo: Es una característica, aspecto de la conducta o una exposición ambiental la cual se asocia con periodontitis destructiva. Su exposición hace que aumente la probabilidad de padecer la enfermedad y su eliminación reduce la posibilidad de adquirirla. Debe de ser biológicamente plausible, y debe demostrarse que precede al desarrollo de la enfermedad en estudios prospectivos.

El tabaco y la diabetes melitus se han descrito como factores de riesgo verdaderos tras amplios debates en la literatura. También se incluyen la placa, microbiota e higiene oral.

Además de los anteriores, se han asociado determinados factores locales como los espacios amplios interproximales y la impactación alimenticia con la perdida de inserción y aumento de sondaje. Del mismo modo se ha asociado la oclusión traumática con una pérdida de hueso alveolar y los hábitos parafuncionales asi como una morfología dentaria específica con un peor pronóstico post-tratamiento periodontal (3).

-Determinantes de riesgo: Son factores de riesgo que no pueden ser modificados: Edad, sexo, raza, genética, nivel socioeconómico. También podemos incluir determinadas enfermedades sistémicas asociadas con déficit o disfunción de los neutrófilos. Se utilizan para identificar grupos de riesgo. Algunas de estas características inmutables no se consideran etiológicas y pueden actuar como factores de confusión (edad, sexo y raza) (4).

-Indicador de riesgo: Es un factor causal biológicamente plausible pero sólo se ha demostrado estar asociado con la enfermedad en estudios transversales y casos-control: estrés, comportamiento, osteopenia y osteoporosis.

En lo que al estrés se refiere, su asociación con la gingivitis ulcero-necrótica se conoce desde hace tiempo, pero se ha demostrado recientemente que tanbién puede haber asociación con periodontitis y gingivitis (5).

Algunos autores incluyen en este apartado la presencia de determinados patógenos como P.g, B.f, P.i y F.n, asi como virus como el Epstein Barr y el Cytomegalovirus y su asociación con la periodontitis $(6,7)$. Aunque estudios longitudinales han demostrado P.g y Espiroquetas como posibles factores de riesgo.

-Predictor de riesgo o marcador de riesgo: Son factores que indican la presencia de la enfermedad y se asocian con un incremento de probabilidad de tener la enfermedad pero no son factores etiológicos. Por ejemplo, altos niveles de PG E2, sangrado al sondaje o el numero de dientes perdidos.

\section{ETIOLOGÍA MULTIFACTORIAL DE LAS ENFERMEDADES PERIODONTALES}

El inicio y progresión de las enfermedades periodontales está influido por una serie de factores locales y sistémicos. Los factores locales incluyen la existencia de enfermedad preexistente, la presencia de áreas de retención de placa y restauraciones defectuosas. Los factores sistémicos pueden tener relación más o menos evidente con la enfermedad periodontal.

Numerosos estudios que examinan los múltiples marcadores y factores potenciales de riesgo, han documentado que la probabilidad de enfermedad grave se ve aumentada por ciertos factores "de fondo":sexo u origen negro o filipino, la edad avanzada, el nivel socioeconómico bajo, ciertas enfermedades sistémi- 
cas (diabetes), el tabaco y la presencia de determinadas bacterias (P.g, P.f, P.i).Se ha observado igualmente que los distintos factores pueden ser importantes según los grupos de población, de donde la raza o edad parecen influir sobre la expresión de la enfermedad (8).

Resumiendo lo anterior, el concepto actual de la etiología multifactorial de las enfermedades periodontales establece que éstas son producidas por una interacción de un agente microbiano único o múltiple considerado como el factor etiológico primario necesario pero no suficiente, un huésped más o menos susceptible y unos factores ambientales que influyen sobre ambos.

\section{FACTORES GENÉTICOS INVOLUCRADOS EN LA ENFERIMEDAD PERIODONTAL}

Odontólogos y científicos tienen hoy día a su disposición una gran riqueza de conocimientos sobre la etiología y patogénesis de las enfermedades periodontales.

El dentista que nota que el patrón dental de un niño se asemeja al de sus padres se preguntará el porqué de este hecho. La respuesta a esta pregunta puede encontrarse en la base epistemológica del mismo que nos proporciona un viaje fascinante a través de la evolución de la ciencia de la célula y el gen.

En el simposium de genética de 1974, se describieron tres métodos que pueden ser aplicados para mejorar el conocimiento de la base genética de las enfermedades periodontales (9). Estos métodos se refieren a estudios en humanos: estudios de gemelos (mono y dicigotos criados juntos o por separados), estudios en familias (con periodontitis de inicio precoz) y estudio de poblaciones (asociación de periodontitis con determinados desórdenes genéticos).

La importancia de la herencia y la genética para la práctica clínica en general y la patología alveolar en particular se destacaron desde muy temprano pues ya desde 1924 Praeger afirmaba que dicho conocimiento podría influenciar la terapia y el pronóstico de las enfermedades orales.

En la magnífica revisión realizada por Thomas M. Hassell, se describe con detalle la evolución a lo largo del siglo XX de la investigación sobre los factores genéticos y la herencia implicados en la enfermedad periodontal. A lo largo de la revisión, y tras el estudio de las numerosas investigaciones llevadas a cabo en gemelos, familias, y poblaciones por diferen- tes autores se corrobora el papel de los factores genéticos en la aparición y desarrollo de la enfermedad periodontal (11).

\section{CONEXIÓN ENTRE EL ANÁLISIS GENÉTICOY LAS ENFERIMEDADES PERIODONTALES}

El estudio genético de una enfermedad es el método que determina la localización cromosómica del gen de un rasgo confirmando así que determinados factores genéticos están involucrados en dicho rasgo.

Está claro que los factores genéticos juegan un papel importante en la susceptibilidad de la enfermedad (12) pero las complejas interacciones que ocurren entre los mecanismos de respuesta del hospedador y la acción de los microorganismos patógenos han hecho que las aclaraciones sobre el papel de los factores genéticos en la enfermedad periodontal sean más difíciles.

La influencia de los factores genéticos en la periodontitis parece ser diferente para los distintos tipos de enfermedad periodontal y ha sido estudiada en cada una de las periodontitis definidas.

\section{PERIODONTITIS AGRESIVAS}

La hipótesis sobre la presencia de factores de riesgo genéticos en el desarrollo de la periodontitis destructiva se originó en principio de la observación de una serie de síndromes que se suponen determinados genéticamente. Las periodontitis prepuberales se han asociado clásicamente con dichos síndromes que predisponen a la destrucción rápida de los tejidos periodontales.

Las que se presentan de forma generalizada se asocian frecuentemente con una disfunción o déficit de neutrófilos lo que causa un reclutamiento local anormal de los neutrófilos y monocitos por consiguiente una respuesta inmune inadecuada.

- En el tipo LAD-1 (deficiencia de adhesión leucocitaria) se observan deficiencias en le subunidad b de las proteínas de membrana MAC-1, LFA-1 y la glicoproteina P150.95 (moléculas que median la adherencia de los neutrófilos a las células endoteliales y la unión al complemento).

- En el tipo LAD-2 se detecta la ausencia del ligando Sialyl-Lewis X (molécula que une a las células endoteliales activadas) (13).

Dichas anomalías se asocian a desórdenes hereditarios como el Síndrome de Chediak-Higashi, Papillon 
Lefévre y neutropenia cíclica y congénita así como a defectos del colágeno como el Síndrome de EhlerDanlos tipo VIII y defectos enzimáticos asociados con la homeostasis del hueso y tejido conectivo como la achalasia e hipofosfatasia.

Se han sugerido una herencia Mendeliana tanto autosómica recesiva como autosómica dominante de periodontitis prepuberales (14). Los factores genéticos tendrían una función modificadora específica junto a los agentes etiológicos primarios (bacterias periodontopáticas) y los factores moderadores del medio ambiente, cuya interacción conduciría a la manifestación clínica de la salud/enfermedad del periodonto (15).

Las periodontitis precoces (prepuberales y juveniles) que no se asocian a síndromes hereditarios son raras, pero se han descrito casos en los cuales se presentan aisladamente. En el caso de las periodontitis juveniles, se han descrito patrones familiares que pueden deberse tanto a causas genéticas como ambientales (transmisión de Actinomyces actinomicetencomitans (Aa) (13). Diversos estudios sugieren que los factores genéticos son responsables en parte de un aumento en la susceptibilidad a este tipo de formas agresivas de enfermedad periodontal y otros descartan que su presencia en miembros de la misma familia se pueda deber solamente a una transmisión de $A a$ (14).

\section{PERIODONTITIS CRÓNICAS}

En general, se considera que hay suficiente base científica a favor de la presencia de factores genéticos en la aparición de periodontitis de aparición temprana. En los adultos, en cambio la evidencia de la participación genética es menos manifiesta. Durante los pasados cinco años, los investigadores han reiniciado la búsqueda de un componente hereditario de la susceptibilidad de la periodontitis del adulto.

Los estudios realizados sobre gemelos sugieren que el papel de la herencia es variable y la evidencia no es clara (13). La identificación de la posible predisposición genética se refiere específicamente a las periodontitis agresivas en pacientes sin enfermedades sistémicas que impliquen una alteración periodontal.

\section{CANDIDATOS GENÉTICOS IMPORTANTES EN LA PERIODONTITIS. POLIMORFISIMOS}

En humanos, los estudios de las variaciones hereditarias del sistema inmune son muy complejas, y el fenotipo observado es frecuentemente el resultado de múltiples influencias genéticas y medioambientales. Esto se observa especialmente en la respuesta del hospedador frente a bacterias Gram negativas y Lipopolisacáridos, en la cual diferentes factores celulares y moleculares entran en juego. Numerosos estudios demuestran cada vez con más fuerza la existencia de determinados polimorfismos genéticos para dichos factores inmunoinflamatorios. Estos afectan tanto la respuesta cuantitativa (defectos estructurales de los genes) como la cualitativa inmunológica del hospedador (polimorfismos regulatorios).

Kornman y cols en 1997 ponen de manifiesto que existe una asociación entre la periodontitis y una variación genética en el gen que codifica la IL-1 sugiriendo que el $30 \%$ de adultos con periodontitis tiene éste genotipo. De este modo, se apunta que la variabilidad clínica que se observa en pacientes periodontales se basa en la influencia de diferentes interleukinas sobre la reabsorción ósea y la destrucción de tejido conectivo. Aquellos pacientes positivos para la IL-l(IL- $1 \alpha / \mathrm{IL}-$ $1 \beta$ ) presentarían elevadas posibilidades de desarrollar periodontitis agresivas (16).

Igualmente se ha sugerido que determinados polimorfismos genéticos se han asociado con altos niveles de TNF- $\alpha$ y Prostaglandinas E2 (estimuladoras de endotoxinas de monocitos) relacionándose con la periodontitis de aparición temprana y la periodontitis del adulto $(17,18,19)$.

Las Prostaglandinas E2 son potentes mediadores inducidos en la respuesta ante diferentes estímulos inmunoinflamatorios (citoquinas, factores de crecimiento y LPS bacterianos), éstas han sido implicadas en periodontitis del adulto así como en periodontitis de aparición temprana como mediadoras de la destrucción de dichas periodontitis.

La IL- 1 y el TNF- $\alpha$ son potentes estimuladores de la reabsorción ósea; una hiperproducción de dichas citoquinas ocasionada por la infección de los patógenos periodontales puede ser uno de los mecanismos de la destrucción de los tejidos periodontales.

Otros estudios de polimorfismos se han llevado a cabo, como el de la interleukina IL-4, potente reguladora de la función de los macrófagos. Se observó que la aparición del polimorfismo para la IL-4 era más frecuente en pacientes con periodontitis agresiva que en los controles (20).

Iguamente, se observó que el polimorfismo para la IL 10 estaba asociado con la periodontitis crónica o agresiva $(21,22,23)$.

La hipótesis de que la variación genética de la expresión de citoquinas pueda ser un importante factor de riesgo para la periodontitis agresiva y crónica está hoy día en voga y a la espera de ser aclarada por 
numerosas investigaciones que se están llevando a cabo.

Como hemos visto, numerosos polimorfismos han sido y continúan siendo investigados como futuros candidatos a factores de riesgo para la susceptibilidad a la enfermedad periodontal. Entre ellos, el fenotipo HLA (Human Leukocite Antigen), que también ha sido investigado como posible factor de resistencia, ha sido asociado fundamentalmente con la periodontitis juvenil y de evolución rápida e incluso con la periodontitis del adulto.

Las moléculas del sistema HLA están presentes en las membranas de las células nucleadas del organismo e intervienen en la respuesta inmune presentando el antígeno a las celulas T; la hipótesis que se han planteado algunos estudios es que la expresión de determinadas moléculas HLA (polimorfismos) pudiese interferir a este nivel.

Hay varios estudios que han encontrado una relación del antígeno HLA-A9 así como el HLA-A28, Bw15, Bw35 y DQwl sugiriéndose que su presencia aumenta la susceptibilidad para las periodontitis agresivas(HLA-A9,-A28,-Bw15) y crónicas(HLA-A9,-A28). Por otro lado, los antígenos HLA se han asociado con la resistencia a determinadas periodontitis agresivas (HLA-A2) y crónicas (HLA-A10,-B5.-A28). Autores como Shapira (1994) concluyen además que determinados antígenos HLA están asociados a una forma localizada de la periodontitis juvenil (HLA A9 y B15) pero no a las formas generalizadas y que ambas son enfermedades diferentes bajo un diferente control genético $(24,25,26,27)$.
Además de ser un eje poco explorado, el campo de estudio del HLA es complejo, la evolución y avance en las técnicas de biología molecular hacen que aparezcan nuevos descubrimientos de antígenos y conceptos lo que conlleva una nueva nomenclatura que obstaculiza aún más la interpretación de numerosos estudios lo que provoca que aparezcan resultados aparentemente divergentes.

Se ha sugerido igualmente que el polimorfismo del gen del receptor de la vitamina D podría estar asociado con una perdida del hueso alveolar y el desarrollo de enfermedad periodontal (28).

Gwin y colaboradores sugirieron tras un estudio la asociación entre el polimorfismo del receptor fMLP y la periodontitis agresiva localizada juvenil (29).

El polimorfismo de los receptores Fc III $\beta$ de los neutrófilos se ha asociado igualmente con la resistencia a periodontitis (30) así como el polimorfismo que define el receptor Fc $\gamma$ RII que ha aparecido en la literatura como un marcador de la susceptibilidad a la periodontitis juvenil localizada (31). Dichos polimorfismos comprometen la comunicación de las células fagocíticas, claves en la respuesta del huésped, ya que son el medio de conexión de las inmunoglobulinas.

Además, se ha observado que el polimorfismo de la $\mathrm{N}$-acetyltransferasa (NAT2) esta significativamente asociado con una perdida de hueso más severa (32).

Basándonos en un esquema publicado por Hart y Kornman , presentamos en la siguiente tabla un resu-

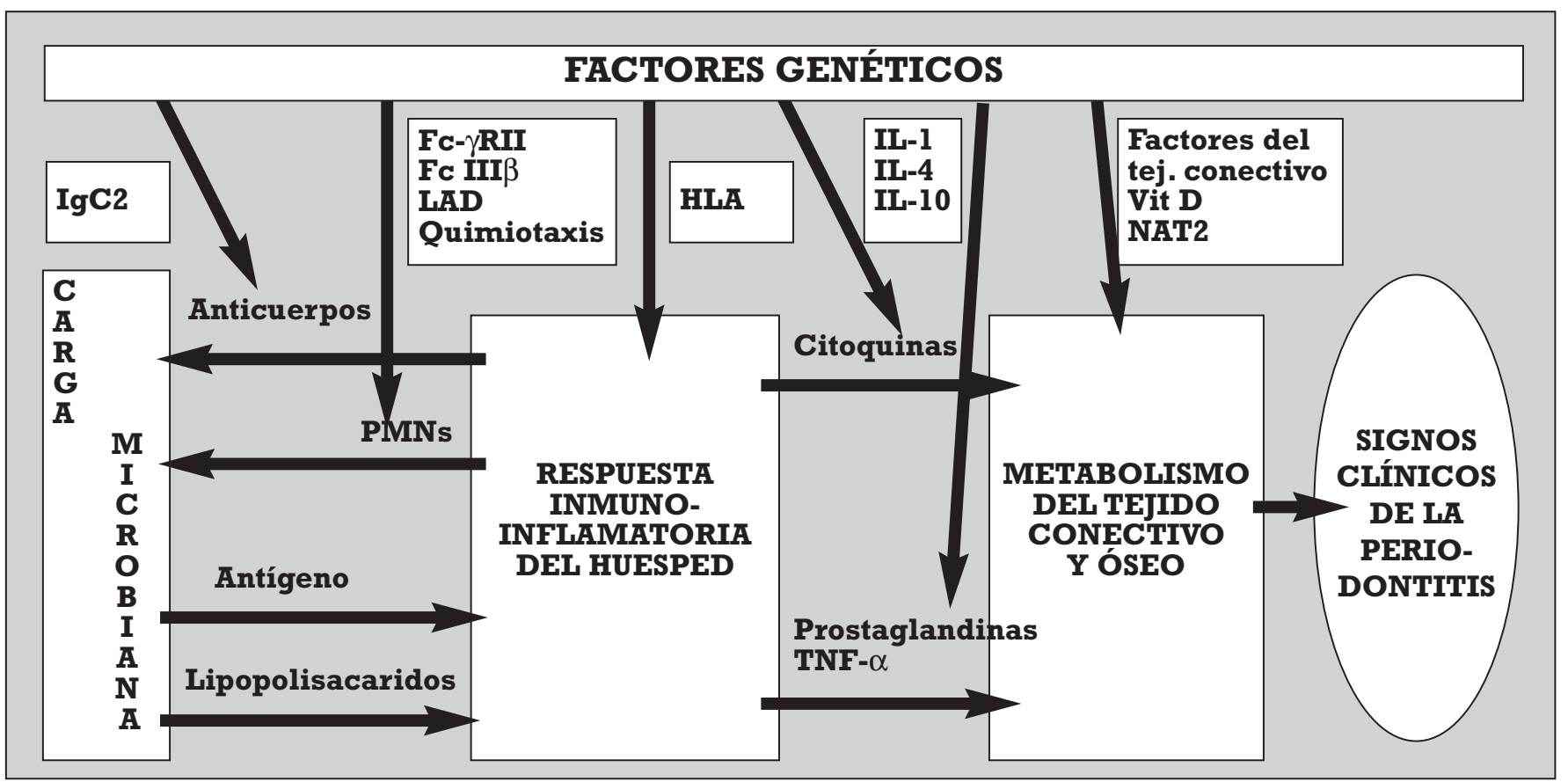


men de la influencia de determinados factores genéticos en la periodontitis y su potencial biológico (33).

\section{ABSTRACT}

Periodontitis is nowadays accepted as a multi-factorial disease with microbial agent as the initiator ,necessary but not enough, and a wide variety of determinants and factors that influence the manifestation and progression of the disease.

The potencial importance of genetics and heredity to the knowledge about the pathogenesis and the clinic dental practice of periodontal diseases has been recognized since the earliest days of dentistry and medicine, but the relative complexity resulting from the interaction among the exposure to oral bacteria and the host response promote the dificulty of the genetics factors role clarification.

Even so, genetic factors influence in periodontal diseases are suggest for several forms of periodontitis. In general, it's considered the existence of sufficient scientific basis in favour of the genetic factors presence in Aggressive Periodontitis, whereas, the evidence of the genetic participation in Chronic Periodontitis isn't so manifest.

A multitude of host factors are involved in responses to microbial challenge and in the subsequent immune responses of the periodontal diseases, so, genetics polymorphisms probably exist in many of if not most of the inflammatory and immune mediators such as demonstrated by the IL-1,IL-4, IL-10, TNF, PGE2, specific HLA antigens (Human Leukocite Antigen), Vitamine D receptor, fMLP receptor, IgG Fc receptors (FcIIIb and FcүRII) and the N-acetyltransferase (NAT2) receptor. Correlation of these genetic polymorphisms with phenotypic characteristics of periodontitis patients groups may provide the frame work for identification of individual risk profiles.

\section{KEY WORDS}

Periodontal diseases. Periodontitis. Risk factors. Genetic Factors.

\section{CONCLUSIONES}

El estudio de los factores de riesgo en la periodontitis, indica el papel imprescindible de las bacterias como factor etiológico de la enfermedad periodontal.
Sin embargo, la susceptibilidad del huésped, en relación con una determinada carga genética, va a determinar las características clínicas de la respuesta del huésped a dicha agresión bacteriana.

El carácter hereditario de las periodontitis es uno de los aspectos más estudiados en los últimos años. Ésta, a parte de ser un proceso multifactorial es una enfermedad multigénica; su desarrollo no depende de un único gen sino de la acción combinada de varios genes y, dependiendo de cuales se encuentren afectados, los polimorfismos que presenten y la población a la que pertenezca un individuo, se presentará un tipo u otro de enfermedad (34).

Las investigaciones que se están llevando a cabo en este campo apuntan hacia una misma dirección: la posibilidad de entendimiento de la etiopatogénia y manejo clínico efectivo de la enfermedad periodontal mediante determinadas pruebas genéticas. Así como la realización de estas pruebas en pacientes periodontales de riesgo puede proporcionar información de cómo va a evolucionar su enfermedad periodontal permitiendo al clínico actuar en la prevención y la evaluación del riesgo en pacientes ya afectados (35). A día de hoy, ya se han elaborado diferentes tests genéticos para evaluar el riesgo periodontal y se ha iniciado su comercialización (Periodontal Susceptibility Test, Interleukin Genetics Inc., Waltham, Mass.) aunque aún no se han tenido resultados concluyentes respecto a su uso (36).

La prevención de una enfermedad común como la enfermedad periodontal es muy complicada a causa de su naturaleza multifactorial que compromete la genética, el medioambiente, el nivel social y otros factores. Por lo tanto, podríamos pensar que la búsqueda de factores de riesgo abre la posibilidad de un mayor éxito en el control y la prevención de las enfermedades periodontales. Desafortunadamente, como expresan Meisel y Kocher en una carta al editor de la revista Eur J Oral Sci, se ha demostrado que para la mayoría de los factores de riesgo de las enfermedades crónicas su utilidad en la predicción de la enfermedad es limitada, además la sensibilidad de dichos factores es a veces pequeña lo que limita la relevancia clínica y su potencial de prevención (37).

\section{BIBLIOGRAFÍA}

1. Delgado M,Doménech JM. Fundamentos de diseño y estadística. UD 7:Investigación científica: Diseño de estudios. Barcelona: Signo; 2005.

2. Genco RJ. Current view of risk factors for periodontal diseases. J Periodontol 1996; 67: 1041-9. 
3. Philstrom BL. Periodontal risk assessement, diagnosis and treatement plannig. Periodontol 2000. 2001; 37-58.

4. Echevarria JJ. Enfermedades periodontales y periimplantarias. Factores de riesgo y su diagnóstico. Av Periodon Implantol. 2003;15: 149-58.

5. Croucher R, Marcenes WS, Torres MS, Hughes F, Sheiham A. The relationship between life-events and periodontitis. A case-control study. J Clin Periodontol. 1997; 24: 3943.

6- Alpagot T, Wolf LF, Smith QT, Tran SD. Risk indicators for periodontal disease in a ratiaclly diverse urban population. J Clin Periodontol 1996; 23: 982-8.

7. Papanou PN. Periodontal diseases: epidemiology. Ann Periodontol 1996; 1: 1-36.

8. Papanau PN, Lindhe J.Epidemiología de la enfermedad periodontal. En:Periodontología clínica e implantología odontológica. Tercera edición.2003; cap.2:69-101.

9. Niswander J. Genetics of common dental disorders. Dent Clin North Am. 1975; 19: 197-206.

10. Schenkein HA. Inheritance as a determinalt susceptibility for periodontitis. J Dent Education. 1998; 62: 840-51.

11. Hassell TM. Genetic influences in caries abd periodontal diseases. Crit Rev Biol Med. 1995; 6; 319-42.

12. Michalowicz BS. Genetic and heritable risk factors in factors in periodontal disease. J Periodontol. 1994; 65:479488.

13. Albandar JM. Global risk factors and risk indicators for periodontal diseases.Periodontol 2000. 2002; 29: 177206.

14.Schenkein HA, Van Dyke TE. Early-onset periodontitis: systemic aspects of etiology and patogénesis. Periodontology 2000. 1994; 6:7-25.

15. Kornman KS, Crane A, Wang $\mathrm{H}-\mathrm{Y}$ et al. The interleukin-1 genotype as a severity factor in adult periodontal disease. J Clin Periodontol. 1997; 24: 72-7.

16. Kornma KS, di Giovine FS. Genetic variations in cytokine expression: a risk factor for severity of adult periodontitis. Ann Periodontol. 1998; 3: 327-38.

17. Shapira L, Stabholz A, Rieckmann P, Kruse N. Genetic polymorphism of the tumor necrosis factor (TNF)-alpha promoter region in families with localizated early-onset periodontitis. J Periodontal Res. 2001; 36: 183-6.

18. Craandijk J, van Krugten MV, Verweij CL, van der Velden
U, Loos BG. Tumor necrosis factor-alpha gene polymorfisms in relation to periodontitis. J Clin Periodontol 2002; 29: 28-34.

19. Michel J, Gonzales JR, Wunderlich D, Diete A, Herrmann JM, Meyle J. Interleukin-4 polymorphisms in early onset periodontitis. J Clin Periodontol. 2001; 28: 483-8.

20. Henning BJ, Parkhill JM, Chapple IL, Heasman PA, Taylor IJ. Dinucleotide repeat polymorphism in the interleukin10 gene promoter (IL-10,G) and genetic susceptibility to early-onset periodontal disease. Genes Immun. 2000; 1: 402-4.

21. Kinane DF, Hodge P, Eskdale J, Ellis R, Gallagher G. Analysis if genetic polymorphisms at the interleukin-10 and tumor necrosis factor loci in early-onset periodontitis. J Periodontal Res. 1999; 34: 379-86.

22. Yamazaki K, Tabeta K, Nakajima T, Ohsawa Y, Ueki K, Itoh $\mathrm{H}$, Yoshie H. Interleukin-10 gene promoter polymorphism in Japanese patients with adult and early-onset periodontitis. J Clin Periodontol. 2001; 28: 828-832.

23. Reinholdt J, Bay I and Svejgaard. Association between HLA-Antigens and periodontal disease. J Dent Res. 1977; 56: 1261-3.

24. Goteiner D and Goldman M. Human Lymphocyte Antigen haplotype and resistance to periodontitis. J Periodontol.1983: 155-7.

25. Amer A, Singh G, Darke C and Dolby E. Association between HLA antigens and periodontal disease. Tissue Antigen. 1988; 31: 53-8.

26. Shapira L, Eizemgerg S, Sela MN, Soskolne A. and Brautbar H. HLA A9 and B15 are associated with generalized form, but not the localized form, of early-onset periodontal diseases.J Periodontol. 1994; 65: 219-23.

27. Hennig BJ, Parkhill JM, Chapple IL, Heasman PA, Taylor IJ. Association of a vitamin D receptor gene polymorphism with localized earl-onset periodontal diseases. J Periodontol 1999; 70: 1032-8.

28. Gwin MR, Sharma A, De Nardin E. Single nucleotide polymorphism of the $\mathrm{N}$-formyl peptide receptor in localized juvenile periodontitis. J Periodontol 1999; 70: 1194-201.

29. Sugita N, KobayashiT, Ando Y, Yoshihara A, Yamamoto K, van de Winkel JG, Miyazaki H, Yoshie H. Increased frecuencia of FcgammaRIIIb-NAl allele in periodontitis resistant subject in elderly Japanese population. J Dent Res. 2001: 80; 914-8.

30. Bredius RGM, de Vries CEE, Troelstra A, van Alphen L, Weening RS, van de Minkel JGJ, Out TA. Phagocytosis os 
Staphylococcus aureus and Haemophilus influenzae type Bna opsonized with polyclonal human IgGl and IgG2 antibodies. Functional hFc-gamma-RIIa polymorphism to IgG2. J Immunol 1993; 151S: 1463-72.

31. Osborne JM, Chacko GW, Barndt JT, Anderson CL. Ethnic variation in frecuency of allelic polymorphism of human Fc-gamma-RIIA determined with allele specific oligonucleotide probes. J Immunol Methids 1994; 173: 207-17.

32. Kocher T, Sawaf H, Fanghanel J, Timm R, Meisel P. Association between bone loss in periodontal disease and polymorphism of $\mathrm{N}$-acetyltransferase (NAT2). J Clin Periodontol. 2002; 29: 21-7.

33. Hart TC, Kornman KS. Genetic factores in the pathogenesis of periodontitis.Periodontology 2000. 1997; 14: 20215.
34. Schenkein, Harvey A. Finding genetic risk factors for periodontal diseases: in the climb worth the view? Periodontology 2000. 2002; 30: 79-90.

35. Donate Elisa, Fias MC, Balda I, Carasol M. El futuro de la odontología dependerá de los avances en genética. Gaceta Dental. 2005; 155:94-98.

36. Greenstein G, Hart TC. Clinical utility of a genetic susceptibility test for severe chronic periodontitis.A critical evaluation. JADA 2002; 133: 452-9.

37. Meisel $P$ y Kocher T. Risk factors on periodontitis and classifying the disease. Letter to the Editor. Eur J Oral Sci. 2003; 111:280-3. 